\title{
Magnetic Properties of $\mathrm{CeNi}_{4} \mathrm{Mn}_{y} \mathrm{Al}_{1-y}$ Compounds
}

\author{
K. SYNORADZKI*AND T. TOLIŃSKI
}

Institute of Molecular Physics, Polish Academy of Sciences, M. Smoluchowskiego 17, 60-179 Poznań, Poland

\begin{abstract}
The magnetic properties of the polycrystalline $\mathrm{CeNi}_{4} \mathrm{Mn}_{y} \mathrm{Al}_{1-y}$ compounds have been investigated combining AC susceptibility, field-cooled and zero-field-cooled DC magnetization and magnetic relaxation measurements. The X-ray diffraction measurements showed that the group $\mathrm{CeNi}_{4} \mathrm{Mn}_{y} \mathrm{Al}_{1-y}$ is isostructural and crystallizes in the $\mathrm{CaCu}_{5}$-type structure $(P 6 / \mathrm{mmm})$. For $0<y<1$ irreversible magnetism, long-time magnetic relaxation effect and evident upshift of the AC susceptibility peak with increasing frequency are observed at low temperatures. The spin-glass-like behaviour originates from disorder due to the statistical occupation of the $3 \mathrm{~g}$ site. Using our data we have constructed the tentative phase diagram.
\end{abstract}

DOI: 10.12693 /APhysPolA.127.210

PACS: 75.50.Lk, 71.20.Lp

\section{Introduction}

Cerium based intermetallic compounds are demonstrative examples of systems with strong electron correlations. They exhibit fascinating effects like: effective mass enhancement (heavy fermions - HF), fluctuating valence $(\mathrm{FV})$, Kondo effect, Kondo lattices (KL) formation, superconductivity, ferromagnetism (FM), spin-glass (SG) behaviour, etc.

Our interest was attracted by the $\mathrm{CeNi}_{4} \mathrm{Al}-\mathrm{CeNi}_{4} \mathrm{Mn}$ isostructural systems due to a possible valence transition and the interplay between $3 d$ and $4 f$ magnetism. The $\mathrm{CeNi}_{4} \mathrm{Mn}$ compound is a soft ferromagnet with $T_{\mathrm{C}} \approx$ $130 \mathrm{~K}$ and a stable $\mathrm{Ce}^{+3}$ valence, whereas in $\mathrm{CeNi}_{4} \mathrm{Al} \mathrm{FV}$ has been observed.

Although many studies have been carried out on $\mathrm{Ce}$ based materials in which the Ce ions are in non-magnetic host environments, very little work has been done on materials in which the Ce ions are embedded in robust internal host magnetic fields [1]. Moreover, the substitution of $\mathrm{Mn}$ by $\mathrm{Al}$ is rather rare.

In this paper, we report for the first time the magnetic properties and we focus on characterization and discussion of the diluted magnetic system of the Mn-doped $\mathrm{CeNi}_{4} \mathrm{Al}(0<y<1)$.

\section{Experimental details}

Polycrystalline samples were prepared by induction melting of stoichiometric amounts of pure elements in the argon atmosphere. The samples were turned and remelted several times to achieve a better homogeneity.

The crystal structure was established by a powder Xray diffraction (XRD) technique, using Co $K_{\alpha}$ radiation. The full-pattern Rietveld refinement using FullProf has confirmed the hexagonal $\mathrm{CaCu}_{5}$-type structure (P6/mmm space group) for all the prepared samples. Ce occupies the $1 \mathrm{a}$ site $(0,0,0)$ and $\mathrm{Ni}(1)$ the $2 \mathrm{c}$ site

\footnotetext{
${ }^{*}$ corresponding author; e-mail: karol.synoradzki@ifmpan.poznan.pl
}

$(1 / 3,2 / 3,0)$. $\mathrm{Ni}(2), \mathrm{Al}$ and $\mathrm{Mn}$ are statistically distributed over the $3 \mathrm{~g}$ sites $(1 / 2,0,1 / 2)$.

The DC and AC magnetic susceptibility were measured in the temperature range $2-300 \mathrm{~K}$ using the PPMS (Quantum Design) commercial device.

\section{Results}

The XRD patterns revealed that all samples show essentially a single phase. Figure 1 presents an exemplary XRD pattern for the $\mathrm{CeNi}_{4} \mathrm{Mn}_{0.7} \mathrm{Al}_{0.3}$ sample recorded at room temperature. The obtained lattice parameters decrease with the Mn content (not shown here). The Vegard law is not strictly obeyed, which may stem from the valence variation of the $\mathrm{Ce}$ and/or Mn atoms. Additionally, the strain energy, the stress field or the compressibility of the components could result in the deviations from the Vegard law. Such variation of lattice parameters are often observed in similar systems $[2,3]$.

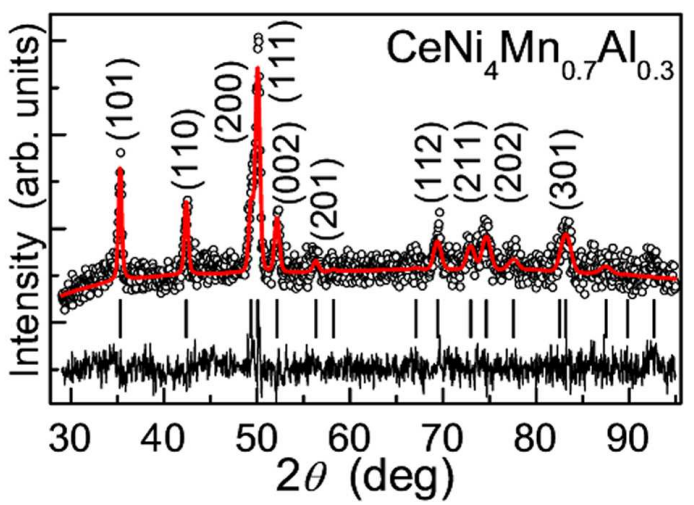

Fig. 1. X-ray diffraction pattern along with the fitting curve for the $\mathrm{CeNi}_{4} \mathrm{Mn}_{0.7} \mathrm{Al}_{0.3}$ sample. The bottom solid line shows the difference between the measured and the calculated patterns. The vertical bars indicate the positions of the structural reflections.

Figure 2 shows the temperature dependence of the zero field cooled (ZFC) magnetization normalized to the maximum value for each sample. There is a peak for all $\mathrm{Mn}$ and Al containing samples, which moves to higher temperature with the $\mathrm{Mn}$ concentration. For the sample with $y=0.1$ the peak is out of temperature range. 
As can be seen in Fig. 3 the peak of $\chi_{\mathrm{DC}}$ becomes broader and moves to lower temperatures while increasing the strength of the magnetic field. Moreover, a comparison of the ZFC and FC curves reveals a strong irreversibility (Fig. 3). Such magnetic behaviour is observed in many $\mathrm{SG}$ systems. Above the peaks, samples are paramagnetic (PM) and the magnetic susceptibility curves can be fitted with the Curie-Weiss formula (not shown here).

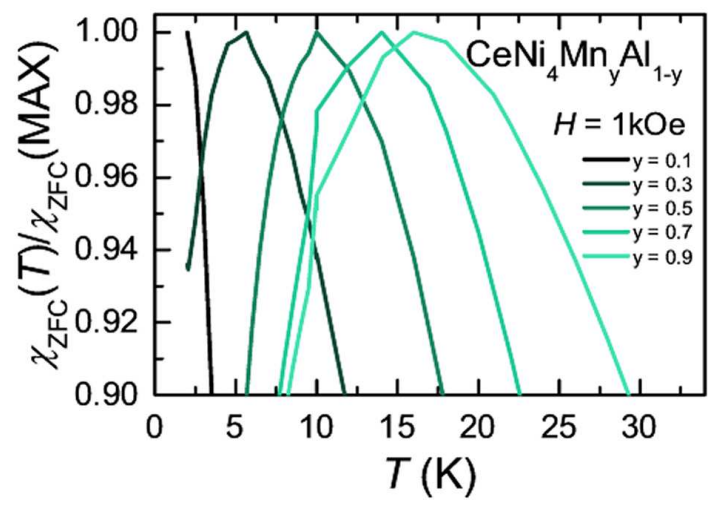

Fig. 2. Temperature dependence (ZFC mode) of the normalized DC magnetization curves for $\mathrm{CeNi}_{4} \mathrm{Mn}_{y} \mathrm{Al}_{1-y}$ as a function of $\mathrm{y}$. The applied magnetic field was equal to $1 \mathrm{kOe}$.

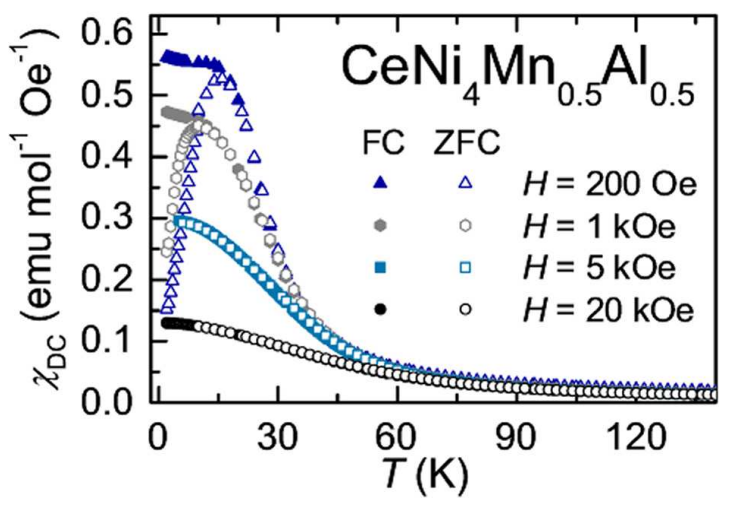

Fig. 3. Temperature variation of the DC magnetization of the $\mathrm{CeNi}_{4} \mathrm{Mn}_{0.5} \mathrm{Al}_{0.5}$ alloy in the $\mathrm{ZFC}$ and FC modes.

The field variation of the magnetizations taken at $2 \mathrm{~K}$ are presented in Fig. 4. For all $\mathrm{Mn}$ and $\mathrm{Al}$ containing samples a hysteresis was observed. One cannot see a complete saturation of magnetization even at high fields for any compositions, which is often observed in SG systems.

In order to confirm the SG effect, we performed an $\mathrm{AC}$ susceptibility $\chi_{\mathrm{AC}}$ measurement at the frequency range $10 \mathrm{~Hz} \leq \omega / 2 \pi \leq 10 \mathrm{kHz}$ with an $\mathrm{AC}$ field $H_{\mathrm{AC}}=5$ Oe. Figure 5 shows real $\chi^{\prime}$ and imaginary $\chi^{\prime \prime}$ part of $\chi_{\mathrm{AC}}$ for $\mathrm{CeNi}_{4} \mathrm{Mn}_{0.5} \mathrm{Al}_{0.5}$ alloy as an example. The position of the cusp in $\chi^{\prime}$ and $\chi^{\prime \prime}$ is frequency-dependent, shifting to higher temperatures at higher frequencies. This behaviour can be described by the empirical Vogel-Fulcher (V-F) law, $f=$ $f_{0} \exp \left(-E_{a} / k_{\mathrm{B}}\left(T_{\mathrm{f}}-T_{0}\right)\right)$, with two fitting parameters:

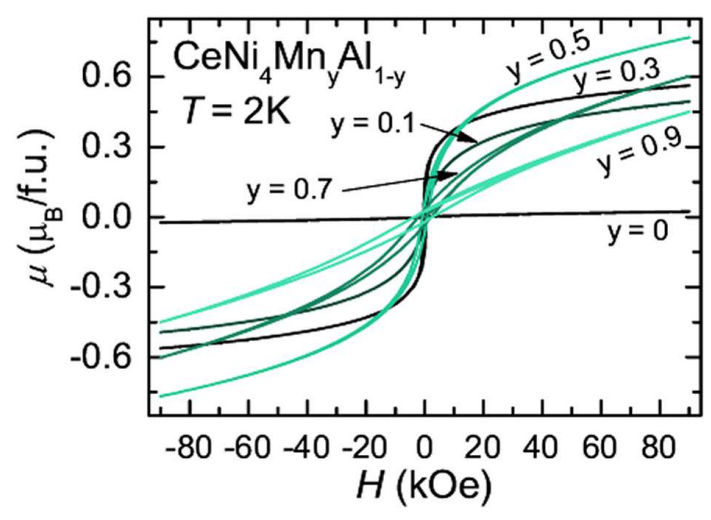

Fig. 4. Isothermal magnetization vs. magnetic field up to $90 \mathrm{kOe}$ measured at $2 \mathrm{~K}$ for the $\mathrm{CeNi}_{4} \mathrm{Mn}_{y} \mathrm{Al}_{1-y}$ alloys.

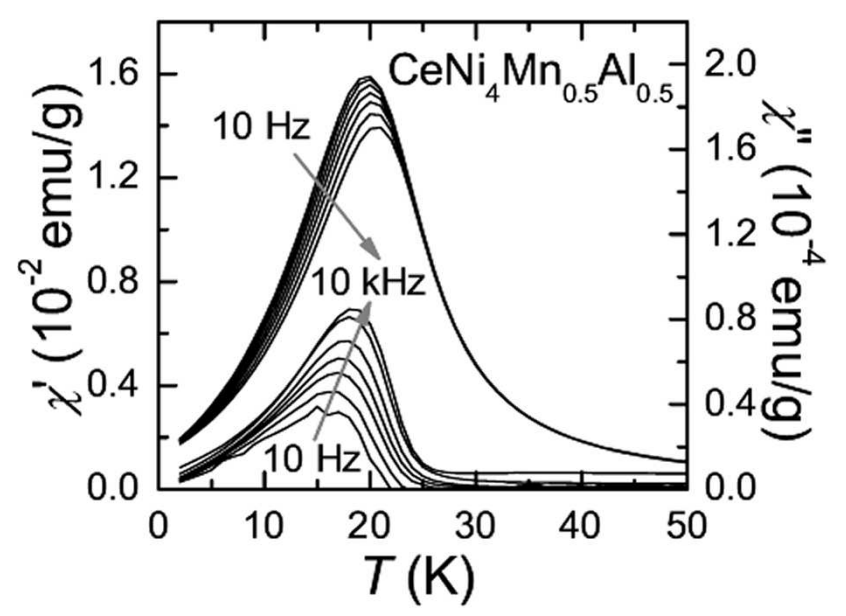

Fig. 5. Temperature and frequency dependence of the real (top, left y-axis) and imaginary (down, right y-axis) part of the $\mathrm{AC}$ magnetization of $\mathrm{CeNi}_{4} \mathrm{Mn}_{0.5} \mathrm{Al}_{0.5}$.

V-F temperature $T_{0}$ and activation energy $E_{a} . f_{0}$ is a characteristic frequency fixed as $10^{13} \mathrm{~Hz}$.

The plot of $T_{\mathrm{f}}$ as a function of $1 / \ln \left(f_{0} / f\right)$ is displayed in Fig. 6a. Additionally, we have used the dynamical slowing down expression, $\tau / \tau_{0}=\left[\left(T_{\mathrm{f}} / T_{\mathrm{g}}\right)-1\right]^{z v}$, to describe the frequency dependence, where $\tau=1 / 2 \pi f, \tau_{0}$ is the relaxation time related to $f_{0}, T_{\mathrm{g}}$ is the spin-glass temperature and $z v$ is the critical exponent, which is in the range 4-12 for SG. From Fig. $6 \mathrm{~b}$ it is clear that this relation is obeyed. Using the analysis method mentioned above, the values of characteristic parameters were estimated for all $\mathrm{Mn}$ and $\mathrm{Al}$ containing samples and all of them fall in the values range typical of SG materials.

The presence of magnetic glassy state is often characterized by the presence of time-dependent remanent magnetism. Unlike a permanent magnet, the magnetization of SG materials decreases fast within a relatively short period. Time dependence of normalized magnetization at $2 \mathrm{~K}$ after cooling the sample without magnetic field is shown in Fig. 7. For all $\mathrm{Mn}$ and $\mathrm{Al}$ containing samples the change of magnetization in time shows similar behaviour. Using a function, $M(T, t)=$ 
$M_{0}(T, 0)+\alpha(T) \ln \left(t+t_{\mathrm{s}}\right)+\beta \exp \left(\left(-t+t_{\mathrm{s}}\right) / \tau\right)$, the $M(t)$ data can been fitted very well over the full time range studied. $M_{0}(T), \tau, t_{\mathrm{s}}, \alpha$ and $\beta$ are fitting parameters.
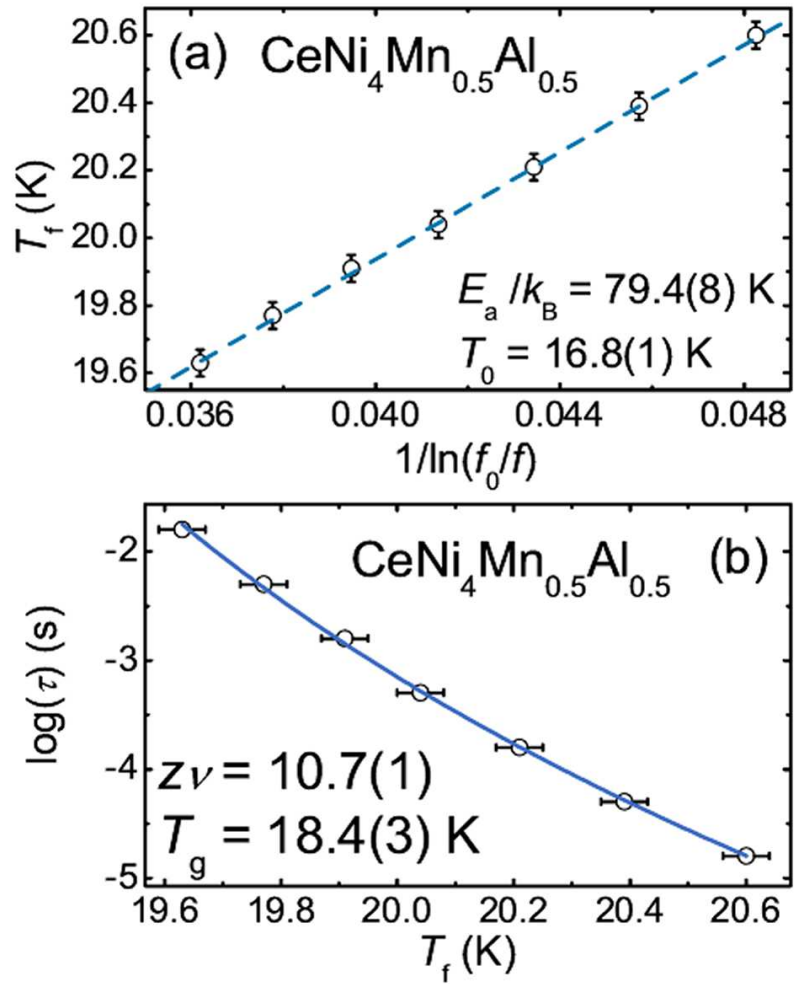

Fig. 6. Frequency dependence of spin freezing temperature $T_{\mathrm{f}}$ for $\mathrm{CeNi}_{4} \mathrm{Mn}_{0.5} \mathrm{Al}_{0.5}$ plotted as (a) $\mathrm{T}_{\mathrm{f}}$ vs. $1 / \ln \left(f_{0} / f\right)$ and $(\mathrm{b}) \log (\tau)$ vs. $T_{\mathrm{f}}$. The solid lines represent the fits to the experimental data.

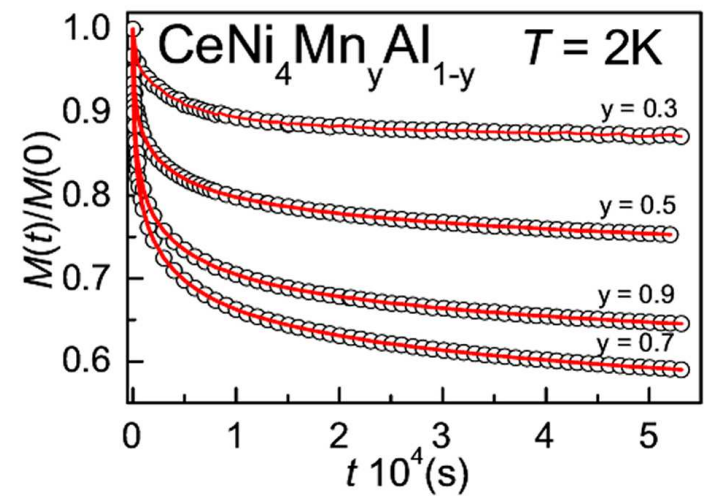

Fig. 7. The magnetic relaxation for the $\mathrm{CeNi}_{4} \mathrm{Mn}_{y} \mathrm{Al}_{1-y}$ samples normalized to magnetization in $t=0$. The solid line is the result of the fitting of the experimental data to $M(T, t)=$ $M_{0}(T, 0)+\alpha(T) \ln \left(t+t_{\mathrm{s}}\right)+\beta \exp \left(\left(-t+t_{\mathrm{s}}\right) / \tau\right)$.

Figure 8 shows a magnetic phase diagram for the $\mathrm{CeNi}_{4} \mathrm{Mn}_{y} \mathrm{Al}_{1-y}$ system where $T_{\mathrm{f}}$ has been obtained both from the $\mathrm{AC}$ and $\mathrm{DC}$ method. In this phase diagram, we define the spin freezing temperature $T_{\mathrm{f}}$ at $f=10 \mathrm{kHz}$ of $\mathrm{CeNi}_{4} \mathrm{Mn}_{y} \mathrm{Al}_{1-y}$ as the peak temperature in $\chi_{\mathrm{AC}}^{\prime}$ curve and as a peak in ZFC $\chi_{\mathrm{DC}}$ measured at $H=1 \mathrm{kOe}$. $T_{\mathrm{f}}$ changes almost linearly, which is often observed in spin glass systems. The deviations for samples with small amount of Mn can be due to the VF of Ce atoms. The magnetic phase diagram shows mostly a simple transition from the paramagnetic state to $\mathrm{SG}$, which is often observed in Mn containing alloys and compounds [2, 4, 5].

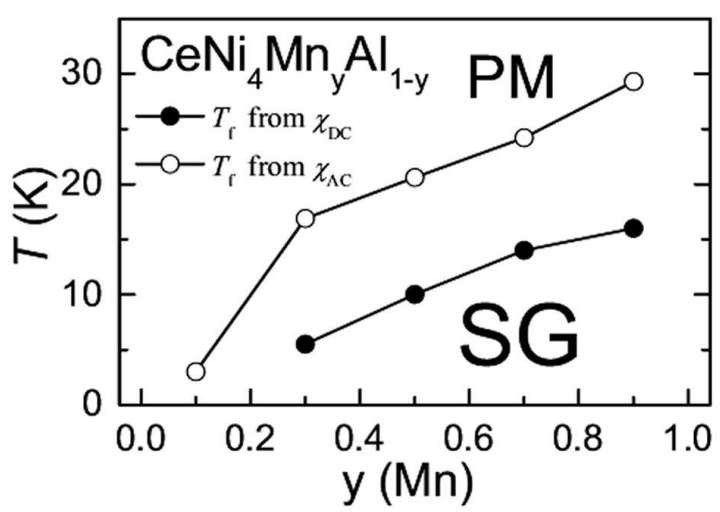

Fig. 8. The magnetic phase diagram for $\mathrm{CeNi}_{4} \mathrm{Mn}_{y} \mathrm{Al}_{1-y}$.

\section{Conclusions}

In conclusion, we have observed the SG behaviour down to $2 \mathrm{~K}$ - the lowest temperature used in our studies, for $0<y<1$ in the $\mathrm{CeNi}_{4} \mathrm{Mn}_{y} \mathrm{Al}_{1-y}$ system, via magnetic properties measurements. Only a small doping with $\mathrm{Mn}$ in the place of $\mathrm{Al}$ is enough to reveal the $\mathrm{SG}$ behaviour. For samples with $y<0.1$ the SG interaction is suppressed, and the system moves towards regime with VF. The origin of SG is due to the magnetic moments on $\mathrm{Mn}$ atoms and due to the disorder on the $3 \mathrm{~g}$ sublattice in the crystallographic unit cell. The statistical distribution of $\mathrm{Mn}, \mathrm{Ni}$ and $\mathrm{Al}$ atoms introduces the randomly distributed magnetic interactions, which is necessary for formation of the frustration in the SG state.

\section{References}

[1] G. Liang, Q. Yao, H. Xi, K. Mochizuki, J.T. Markert, M. Croft, J. Alloys Comp. 414, 8 (2006).

[2] K. Synoradzki, T. Toliński, J. Phys. Condens. Matter 24, 136003 (2012).

[3] G. Liang, F. Yen, J. Appl. Phys 103, 07 B719 (2008).

[4] T. Toliński, K. Synoradzki, Intermetallics 19, 62 (2011).

[5] X. Liu, S. Matsuishi, S. Fujitsu, H. Hosono, Phys. Rev. B 84, 214439 (2011). 